\title{
Herpetofauna do Parque Natural Municipal de Sertão, Rio Grande do Sul, Brasil
}

\author{
Noeli Zanella ${ }^{1,4}$, Almir de Paula ${ }^{2}$, Samara Arsego Guaragni ${ }^{3}$ \& Leonardo de Souza Machado \\ ${ }^{1}$ Instituto de Ciências biológicas, Universidade de Passo Fundo-UPF, \\ CP 611/631, CEP 99001-970, Passo Fundo, RS, Brasil \\ ${ }^{2}$ Laboratório de Herpetologia, Departamento de Zoologia, Instituto de Biologia, \\ Universidade de Brasília - UnB, Campus Darcy Ribeiro, CEP 70910-900, Brasília, DF, Brasil \\ ${ }^{3}$ Departamento de Zoologia, Instituto de Biociências, \\ Universidade Federal do Rio Grande do Sul-UFRGS, Av. Bento Gonçalves, 9.500, \\ Agronomia, CEP 91501-970, Porto Alegre, RS, Brasil \\ ${ }^{4}$ Autor para correspondência: Noeli Zanella,e-mail: zanella@upf.br
}

ZANELlA, N., PAULA, A., GUARAGNI, S.A. \& MACHADO, L.S. Herpetofauna of the Parque Natural Municipal de Sertão, Rio Grande do Sul, Brazil. Biota Neotrop. 13(4): http://www.biotaneotropica.org.br/ v13n4/en/abstract?inventory+bn03113042013

Abstract: The northern part of the state of Rio Grande do Sul was extensively deforested in recent decades, and only isolated fragments of the original vegetation remain. The Parque Natural Municipal de Sertão comprises a remnant of Atlantic Rain Forest, one of the largest fragments in northern Rio Grande do Sul. This study examined the diversity of the herpetofauna, focusing on environmental distribution, seasonality and species richness. We used complementary methods of sampling such as pitfall traps, active search and occasional encounters. We recorded 23 species of frogs, nine snakes and one lizard. With respect to the relationship between amphibians and reptiles and the structural and abiotic factors of the environment, the edge of the fragment showed higher richness and increased dominance of amphibians and reptiles than the interior. The highest amphibian abundances were found when the minimum temperatures were higher and maximum temperatures were lower. The abundance of snakes was correlated with humidity, and species richness was influenced by humidity and monthly average temperature. Our results reinforce the importance of the conservation of forested areas to the maintenance of the species Keywords: anuran, community, conservation, reptile, faunistic inventory, diversty.

ZANElla, N., PAUlA, A., GUARAGNI, S.A. \& MACHADO, L.S. Herpetofauna do Parque Natural Municipal de Sertão, Rio Grande do Sul, Brasil. Biota Neotrop. 13(4): http://www.biotaneotropica.org.br/ v13n4/pt/abstract?inventory+bn03113042013

Resumo: O norte do Rio Grande do Sul foi extensivamente desflorestado durante as últimas décadas e restaram somente fragmentos isolados das áreas originais. O Parque Natural Municipal de Sertão compreende um remanescente de Mata Atlântica, um dos maiores fragmentos do norte do Rio Grande do Sul. O objetivo deste estudo foi conhecer a diversidade da herpetofauna, enfocando aspectos da distribuição no ambiente, sazonalidade e riqueza de espécies. Utilizamos métodos complementares como armadilhas de interceptação e queda, procura ativa e encontros ocasionais. Registramos 23 espécies de anfíbios anuros, nove de serpentes e uma de lagarto. Encontramos relação entre os anfíbios e répteis e os fatores estruturais e abióticos do ambiente, onde a borda do fragmento apresentou maior riqueza e maior dominância de anfíbios e répteis que o interior do fragmento. As maiores abundâncias de anfíbios foram encontradas quando as temperaturas mínimas eram maiores e temperaturas máximas menores. A abundância de serpentes foi correlacionada com a umidade e a riqueza foi influenciada pela umidade e temperatura média mensal. Nossos resultados reforçam a importância da conservação de áreas florestadas para a manutenção das espécies.

Palavras chaves: anuros, comunidades, conservação, répteis, inventário faunistico, diversidade. 


\section{Introdução}

A Mata Atlântica é um dos 34 hotspots mundiais de biodiversidade, abrigando mais de 8.000 espécies endêmicas (Myers et al. 2000, Mittermeier et al. 2004). O processo de fragmentação da Mata Atlântica, incluindo a Floresta Ombrófila Mista, originou a fragmentação progressiva de remanescentes de florestas naturais em pequenas manchas, isoladas por plantações ou pelo desenvolvimento industrial ou urbano. Dentre as consequências mais severas deste processo estão a alteração da riqueza e composição das espécies (Mähler Junior \& Larocca 2009).

O Brasil é o país com a maior diversidade de anfíbios do planeta, com cerca de 946 espécies (Segalla et al. 2012) e o terceiro maior em riqueza de répteis, com aproximadamente 732 espécies (Bérnils $\&$ Costa 2011). Entretanto, foram registradas aproximadamente 94 espécies de anfíbios e 79 de serpentes para o Rio Grande do Sul (Universidade... 2010), sendo importante a ampliação do conhecimento do grupo.

Devido às suas características físicas e fisiológicas, anfíbios e répteis são organismos sensíveis à alterações nos hábitats e estudos de distribuição espacial e temporal das espécies tem revelado que fatores bióticos e abióticos exercem influência sobre o grupo, contribuindo para a estruturação das comunidades (Toledo et al. 2003, Moreira et al. 2008, Santos et al. 2008, Narvaes et al. 2009, Bertoluci et al. 2009, Armstrong \& Conte 2010, Vasconcelos et al. 2010, Lucas \& Marocco 2011, Silva et al. 2011). Embora estudos recentes de ecologia estejam contribuindo para o conhecimento dos anfíbios do Rio Grande do Sul (Both et al. 2008, Maltchik et al. 2008, Moreira et al. 2008, Santos et al. 2008), muitos aspectos permanecem com poucas informações. No norte do Rio Grande do Sul os estudos de herpetofauna em remanescentes florestais são escassos (Zanella \& Cechin 2006), e por esse motivo, ampliar estudos são de extrema importância para o conhecimento da diversidade e conservação das espécies da região.

Este estudo tem por objetivo fornecer uma lista de espécies de anfíbios e répteis da região norte do Rio Grande do Sul, verificando a influência de variáveis climáticas sobre a riqueza e abundância das espécies, utilizando metodologias associadas.

\section{Material e Métodos}

\section{1. Área de estudo}

O Parque Natural Municipal de Sertão (PNMS) (28 02’ 31' S e $52^{\circ} 13^{\prime} 28^{\prime}$ 'W), localizado no Município de Sertão, norte do estado do Rio Grande do Sul (RS), configura-se como um remanescente de Mata Atlântica (Figura 1). Possui aproximadamente 500 ha, com florestas de transição entre os pinhais dos Campos de Cima da Serra e as florestas da bacia do Uruguai. Apresenta uma vegetação mista denominada Floresta Ombrófila Mista (Quadros \& Pillar 2002), contendo em seus estratos inferiores vegetação característica do Planalto e da bacia do Alto Uruguai. A região possui um gradiente de altitude que varia entre 630 a $740 \mathrm{~m}$ e um clima definido como subtropical úmido, com a temperatura média do mês mais quente superior a $22{ }^{\circ} \mathrm{C}$ e temperaturas mínimas com média de $13,2^{\circ} \mathrm{C}$. As chuvas são bem distribuídas, com média de $1.787,8 \mathrm{~mm}$ por ano. A umidade do ar média é de 72\% (Embrapa 2011).

Atividades econômicas têm causado a fragmentação da Floresta Ombrófila Mista, que na maioria das vezes, é substituída por pastagens, monocultivos e plantios homogêneos de espécies arbóreas exóticas (Mähler Junior \& Larocca 2009). Esse processo de substituição da vegetação natural por cultivo de monoculturas tem sido observado no entorno do Parque e tem contribuído para a rápida degradação da área de estudo.

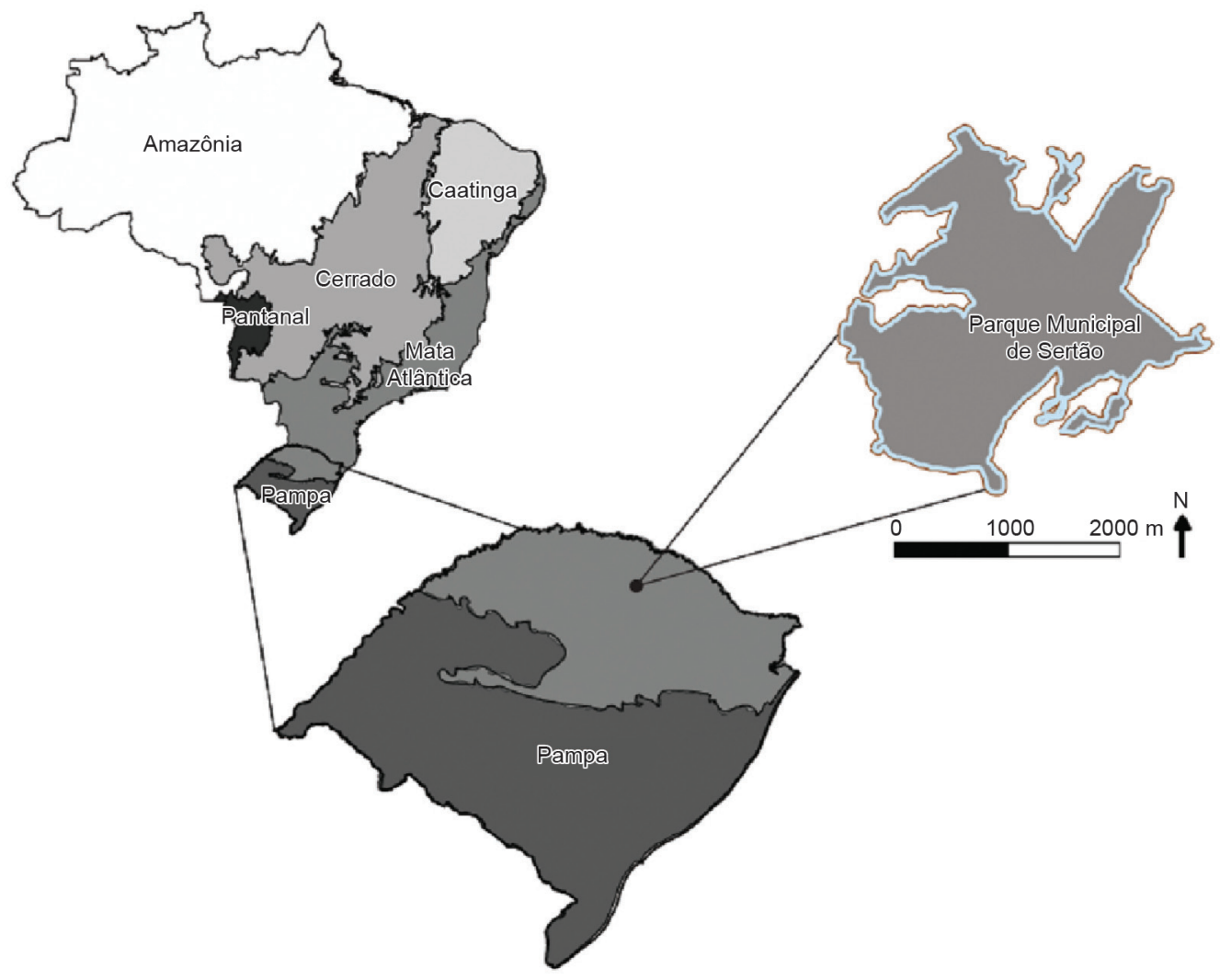

Figura 1. Localização da área de estudo, município de Sertão, Rio Grande do Sul, Brasil.

Figure 1. Location of the study area, municipality of Sertão, Rio Grande do Sul, Brazil. 


\section{Amostragem da herpetofauna}

Amostramos os anfíbios e répteis entre maio de 2008 a abril de 2010 de forma sistematizada e até janeiro de 2011 com procura adicional. Utilizamos três métodos de amostragens: (1) Armadilhas de interceptação e queda (Cechin \& Martins 2000) (AIQ), instaladas em quatro parcelas diferentes na área. Cada uma das parcelas era composta de oito recipientes com capacidade para $150 \mathrm{~L}$, totalizando 32 recipientes enterrados, que foram dispostos em linha reta dentro da mata, com uma distância de $10 \mathrm{~m}$ entre si. Duas séries de armadilhas foram instaladas em área de borda de mata e duas em interior do fragmento, transpassadas por uma tela de aproximadamente $1 \mathrm{~m}$ de altura. As armadilhas permaneceram abertas por 24 meses (720 dias e $17.280 \mathrm{~h}$ ) e revisadas duas a três vezes por semana. Exemplares testemunho foram coletados e depositados nas Coleções de Anfíbios e Répteis da Universidade de Passo Fundo. (2) Na procura ativa (PA), percorremos trilhas em ambientes naturais, no interior de mata, em banhados e áreas do entorno do fragmento, inspecionando tocas, troncos e pedras durante o dia e a noite e também realizamos a escuta de vocalizações para anfíbios. A equipe era composta de uma a cinco pessoas, totalizando 561 horas/observador. (3) Encontros ocasionais e equipe (EO/EE), que consistiu no encontro de anfíbios e répteis por moradores próximos ao fragmento florestal, que receberam um recipiente com formol 10\% para acomodar o material coletado, e os encontros pela equipe durante o deslocamento pela mata ou áreas de entorno, quando não faziam parte de PA.

\section{Análise de dados}

Para analisar a riqueza das espécies coletadas nas armadilhas de queda, utilizamos os estimadores não-paramétricos Bootstrap e Jacknife de primeira ordem e curvas de rarefação de espécies (Colwell \& Coddington 1994), com 1000 aleatorizações. Para isso, cada mês foi considerado uma unidade amostral, resultando em 24 amostras para anfíbios e répteis.
Analisamos a dominância das espécies entre os ambientes amostrados, considerando a borda (L1 e L4) e o interior do fragmento (L2 e L3), utilizando o Índice de Berger-Parker (Magurran 1988). Este índice considera a maior proporção da espécie com maior número de indivíduos. Foi utilizado o teste $t$ para verificar se havia diferença na abundância de espécies entre os dois ambientes. Também utilizamos o índice de equitabilidade Hill (modificado) que se refere à distribuição da abundância das espécies em uma amostra com a finalidade de averiguar se são igualmente abundantes. Verificamos a influência das variáveis climáticas (temperaturas máxima, média e mínima mensais, pluviosidade média mensal e umidade média mensal) sobre a riqueza e abundância dos anfíbios e répteis coletados apenas nas armadilhas de queda. Para isso, realizamos uma análise de autocovariância para confirmar a independência de nossas amostragens mensais. Padronizamos as variáveis quantitativas temporais para testar a associação entre as variáveis climáticas e a riqueza e abundância de anfíbios e répteis e realizamos uma regressão múltipla por passos, com seleção manual de modelos, contendo apenas a interseção, e então verificamos se novas variáveis poderiam ser incluídas e, posteriormente, excluídas do modelo, sem perda de informação, através do teste do qui-quadrado (Tabachnick \& Fidell 2007). Todas as análises foram realizadas no programa $\mathrm{R}$ ( $\mathrm{R}$ Development Core Team 2011), com o nível de significância de 5\%.

As variáveis climáticas foram obtidas da base de dados da Embrapa Trigo de Passo Fundo (http://www.cnpt.embrapa.br).

\section{Resultados}

Registramos 23 espécies de anuros no Parque Natural Municipal de Sertão, distribuídas em sete famílias: Brachycephalidae $(n=1)$, Bufonidae $(n=3)$, Cycloramphidae $(n=2)$, Hylidae $(n=8)$, Leiuperidae $(n=4)$, Leptodactylidae $(n=4)$ e Microhylidae $(n=1)$ (Tabela 1). Armadilhas de interceptação e queda capturaram $82,6 \%(n=19)$ do total de espécies da área de estudo, procura ativa amostrou $65,2 \%$

Tabela 1. Riqueza de espécies de anuros, metodologia aplicada e área de amostragem das espécies no Parque Natural Municipal de Sertão, no período de maio de 2008 a abril de 2010.

Table 1. Anuran species richness, applied methodology and sampling area in the Parque Natural Municipal de Sertão, from May 2008 through April 2010.

\begin{tabular}{|c|c|c|c|c|}
\hline Família & $\begin{array}{c}\text { Espécie } \\
\end{array}$ & Metodologia & Interior & Borda \\
\hline Brachycephalidae & Ischnocnema henselii (Peters, 1872) & $\mathrm{AIQ} / \mathrm{EO}$ & $\mathrm{x}$ & $\mathrm{x}$ \\
\hline \multirow{3}{*}{ Bufonidae } & Melanophyniscus devincenzii Klappenbach, 1968 & $\mathrm{AIQ} / \mathrm{EO} / \mathrm{PA}$ & $\mathrm{x}$ & $\mathrm{x}$ \\
\hline & Rhinella henseli (A. Lutz, 1934) & AIQ & $\mathrm{x}$ & $\mathrm{x}$ \\
\hline & Rhinella icterica (Spix, 1824) & $\mathrm{AIQ} / \mathrm{PA}$ & $\mathrm{x}$ & $\mathrm{x}$ \\
\hline \multirow{2}{*}{ Cycloramphidae } & Odontophrynus americanus (Duméril \& Bibron, 1841) & AIQ & $\mathrm{x}$ & $\mathrm{x}$ \\
\hline & Proceratophrys bigibbosa (Peters, 1872) & AIQ/PA & $\mathrm{x}$ & $\mathrm{x}$ \\
\hline \multirow{8}{*}{ Hylidae } & Aplastodiscus perviridis A. Lutz, 1950 & AIQ/PA & $\mathrm{x}$ & $\mathrm{x}$ \\
\hline & Dendropsophus minutus (Peters, 1872) & PA & & $\mathrm{x}$ \\
\hline & Hypsiboas curupi Garcia, Faivovichi \& Haddad, 2007 & $\mathrm{AIQ} / \mathrm{PA}$ & $\mathrm{x}$ & $\mathrm{x}$ \\
\hline & Hypsiboas faber (Wied-Neuwied, 1821) & AIQ/PA & $\mathrm{x}$ & $\mathrm{x}$ \\
\hline & Hypsiboas pulchellus (Duméril \& Bibron, 1841) & PA & & $\mathrm{x}$ \\
\hline & Scinax aromothyella Faivovich, 2005 & AIQ & & $\mathrm{x}$ \\
\hline & Scinax fuscovarius (A. Lutz, 1925) & AIQ/PA & $\mathrm{x}$ & $\mathrm{x}$ \\
\hline & Scinax squalirostris (A. Lutz, 1925) & PLT & & $\mathrm{x}$ \\
\hline \multirow{4}{*}{ Leiuperidae } & Physalaemus biligonigerus (Cope, 1861) & AIQ & & $\mathrm{x}$ \\
\hline & Physalaemus cuvieri Fitzinger, 1826 & $\mathrm{AIQ} / \mathrm{PA}$ & $\mathrm{x}$ & $\mathrm{x}$ \\
\hline & Physalaemus gracilis (Boulenger, 1883) & AIQ/PA & $\mathrm{x}$ & $\mathrm{x}$ \\
\hline & Physalaemus lisei Braun \& Braun, 1977 & AIQ & & $\mathrm{x}$ \\
\hline \multirow{4}{*}{ Leptodactylidae } & Leptodactylus fuscus (Schneider, 1799) & PA & & $\mathrm{x}$ \\
\hline & Leptodactylus latrans (Steffen, 1815) & AIQ/PA & & $\mathrm{x}$ \\
\hline & Leptodactylus mystacinus (Burmeister, 1861) & AIQ & $\mathrm{x}$ & $\mathrm{x}$ \\
\hline & Leptodactylus plaumanni Ahl, 1936 & $\mathrm{AIQ} / \mathrm{PA}$ & $\mathrm{x}$ & $\mathrm{x}$ \\
\hline Microhylidae & Elachistocleis bicolor (Guérin-Menéville, 1838) & $\mathrm{AIQ} / \mathrm{PA}$ & $\mathrm{x}$ & $\mathrm{x}$ \\
\hline
\end{tabular}

\footnotetext{
Método de amostragem: $\mathrm{PA}=$ procura ativa, $\mathrm{EO}=$ encontro ocasional, $\mathrm{AIQ}=$ armadilha de interceptação e queda.
}

Sampling method: $\mathrm{PA}=$ active search, $\mathrm{EO}=$ occasional encounter, $\mathrm{AIQ}=$ pitfall traps. 
Tabela 2. Riqueza de répteis, metodologia aplicada e área de amostragem das espécies no Parque Natural Municipal de Sertão, no período de maio de 2008 a abril de 2010 .

Table 2. Reptile species richness, applied methodology and sampling area in the Parque Natural Municipal de Sertão from May 2008 through April 2010.

\begin{tabular}{|c|c|c|c|c|}
\hline Família & Espécie & Tipo de registro & Interior & Borda \\
\hline \multirow{7}{*}{ Dipsadidae } & Atractus paraguayensis Werner, 1924 & AIQ & $\mathrm{x}$ & $\mathrm{x}$ \\
\hline & Echinanthera cyanopleura (Cope, 1885) & AIQ & $\mathrm{x}$ & $\mathrm{x}$ \\
\hline & Oxyrhopus clathratus Duméril, Bibron \& Duméril, 1854 & AIQ/PA & $\mathrm{x}$ & $\mathrm{x}$ \\
\hline & Philodryas aestiva (Duméril, Bibron \& Duméril, 1854) & EO & & $\mathrm{x}$ \\
\hline & Taeniophallus affinis (Günther, 1858) & AIQ & & $\mathrm{x}$ \\
\hline & Tomodon dorsatus Duméril, Bibron \& Duméril, 1854 & AIQ/PA & $\mathrm{x}$ & $\mathrm{x}$ \\
\hline & Xenodon merremii (Wagler, 1824) & $\mathrm{EO}$ & & $\mathrm{x}$ \\
\hline \multirow{2}{*}{ Viperidae } & Bothrops cotiara (Gomes, 1913) & AIQ & $\mathrm{x}$ & \\
\hline & B. jararaca (Wied, 1824) & AIQ/PA & $\mathrm{x}$ & $\mathrm{x}$ \\
\hline Teiidae & Salvator merianae Duméril \& Bibron, 1839 & EO & $\mathrm{x}$ & $\mathrm{x}$ \\
\hline
\end{tabular}

Método de amostragem: $\mathrm{PA}$ = procura ativa, $\mathrm{EO}$ = encontro ocasional, $\mathrm{AIQ}$ = armadilha de interceptação e queda.

Sampling method: $\mathrm{PA}=$ active search, $\mathrm{EO}=$ occasional encounter, $\mathrm{AIQ}=$ pitfall traps.

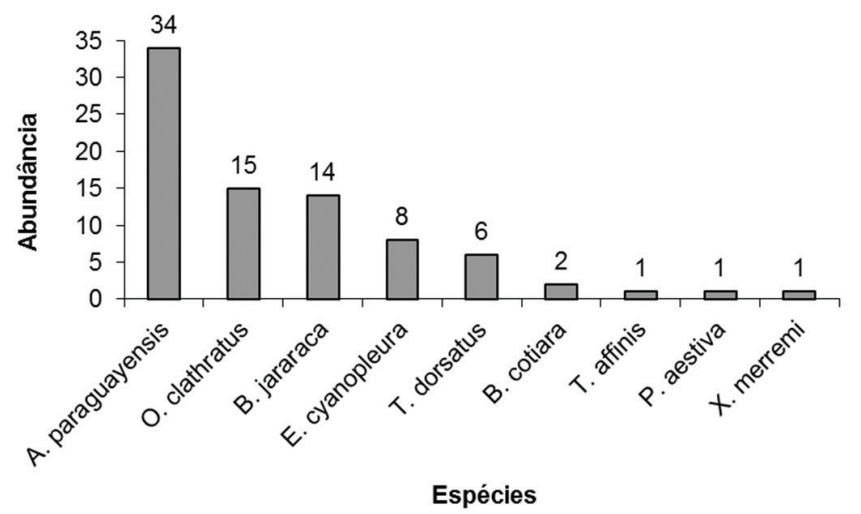

Figura 2. Abundância de serpentes utilizando todos os métodos no Parque Natural Municipal de Sertão, no período de maio de 2008 a abril de 2010.

Figure 2. Abundance of snakes in the Parque Natural Municipal de Sertão from May 2008 through April 2010, using all methods.

$(n=15)$ e os encontros ocasionais amostraram $8,9 \%(n=2)$. Seis espécies foram exclusivas das armadilhas de queda (26\%) e três de procura ativa (13\%) (Tabela 1). Para répteis, registramos 10 espécies, sendo nove serpentes pertencentes a duas famílias: Colubridae $(n=7)$ e Viperidae $(\mathrm{n}=2)$ e uma espécie de lagarto (Teiidae) (Figura 2, Tabela 2). Cerca de $70 \%(n=7)$ das espécies foram coletadas utilizando armadilhas de interceptação e queda. Amostramos três espécies de serpentes (Bothrops jararaca, Oxyrhopus clathratus e Tomodon dorsatus) utilizando procura ativa, as quais também foram encontradas em armadilhas de interceptação e queda e Philodryas aestiva, Xenodon merremii e Salvator merianae foram registradas somente em encontros ocasionais. Atractus paraguayensis, B. cotiara, Echinanthera cyanopleura e Taeniophallus affinis foram amostradas exclusivamente com armadilhas de interceptação e queda.

A borda do fragmento apresentou maior riqueza $(n=19)$ e maior dominância de anfíbios $\left(\mathrm{D}_{\mathrm{bp}}=0.44\right)$ (Figura $3 \mathrm{~A}$ ) que o interior do fragmento $\left(\mathrm{n}=16 ; \mathrm{D}_{\mathrm{bp}}=0.33\right)$ (Figura $\left.3 \mathrm{~B}\right)$. Physalaemus gracilis foi dominante nas duas áreas, representando $44,3 \%$ dos registros para a borda e $32,9 \%$ para o interior. Para répteis também ocorreram variações entre as áreas e espécies dominantes, sendo que a borda do fragmento apresentou maior riqueza $(n=7)$ e maior dominância $\left(D_{b p}=0.54\right)$ que o interior do fragmento $\left(n=6 ; D_{b p}=0.31\right)$. A espécie dominante nos dois ambientes foi Atractus paraguayensis, representando $53,8 \%$ dos registros na borda e $31,2 \%$ no interior. A equitabilidade de anfíbios variou na borda $\left(\mathrm{E}_{\mathrm{H}}=2,51\right)$ e no centro $\left(E_{H}=3,15\right)$ assim como das serpentes (borda: $E_{H}=2,53$; centro=4,06).

As curvas de rarefação da riqueza para anfíbios, utilizando armadilhas de interceptação e queda, não estabilizaram (Bootstrap=20.53 $\pm 0,75$; Jacknife 1=22.83 $\pm 2,99$ ) (Figura 4A). Para répteis, utilizando armadilhas, as curvas de rarefação de riqueza, tenderam à estabilização (Bootstrap $=9,86 \pm 0,35$; Jacknife $1=10,92 \pm 1,33$ ), indicando que utilizando essas metodologias, ainda poderiam ser coletadas mais espécies na área de estudo (Figura 4B).

$\mathrm{O}$ melhor modelo selecionado por passos para a abundância de anfíbios $\left(\mathrm{F}=4,85 ; \mathrm{df}=21 ; \mathrm{r}^{2}=0,32 ; \mathrm{P}=0,02\right)$, revelou que existe influência positiva da temperatura mínima mensal e negativa da temperatura máxima mensal (Tabela 3). Isso indica que maiores abundâncias de anfíbios foram encontradas em meses de maiores temperaturas mínimas e menores temperaturas máximas. Já para a riqueza de anfíbios, não houveram variáveis climáticas selecionadas no modelo testado. A abundância de serpentes foi negativamente relacionada com a variável umidade relativa do ar $(\mathrm{F}=4,14 ; \mathrm{df}=22$; $\left.\mathrm{r}^{2}=0,16 ; \mathrm{P}=0,05\right)$, o que revela que foram amostradas mais serpentes em meses com menor umidade relativa do ar. Para a riqueza de serpentes a umidade relativa do ar influenciou negativamente e a temperatura média mensal positivamente $\left(\mathrm{F}=11,18 ; \mathrm{df}=21 ; \mathrm{r}^{2}=0,52\right.$; $\mathrm{P}<0,001$; Tabela 3 ), o que indica que os meses com menor umidade relativa do ar e maior temperatura foram os que apresentaram maior riqueza de serpentes no Parque Natural Municipal de Sertão.

\section{Discussão}

A riqueza de anfíbios encontrada no Parque Natural Municipal de Sertão é compatível com as espécies ocorrente na Floresta Ombrófila Mista. Em estudo realizado em um remanescente em Santa Catarina, $47,2 \%$ da anurofauna nativa foi semelhante, indicando que a riqueza se aproxima de outros remanescentes deste tipo de floresta no sul do Brasil (Lucas \& Marocco 2011). A predominância da família Hylidae sobre as demais é um padrão comum na região neotropical, tanto em formações abertas como em ambientes florestais (Duellman 1999), como já verificado em diversas comunidades de anuros (Pombal Junior \& Gordo 2004, Ávila-Ferreira \& Ferreira 2004, Vasconcelos \& Rossa-Feres 2005, Conte \& Rossa-Feres 2006, Bertoluci et al. 2007, Santos et al. 2008, Lucas \& Fortes 2008, Bertoluci et al. 2009, Narvaes et al. 2009, Lucas \& Marocco 2011). A ausência de outras espécies que poderiam ocorrer em floresta de araucária pode ter sido influenciada pelo tipo de amostragem que, basicamente neste estudo, foi por armadilhas de interceptação e queda, que não 


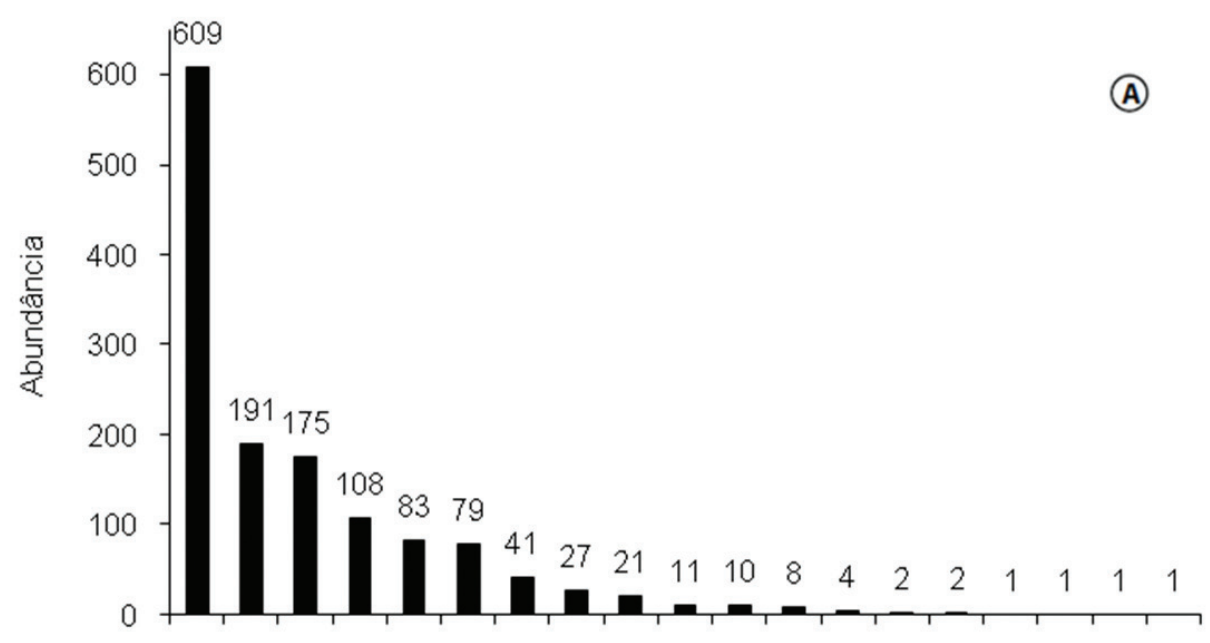

$\mathrm{Pg}$ Ih Lp Pb Rh Ri Md Pc Lm Eb Ap Oa LI Pbi Sf Hf Hc Sa Ss

Espécies

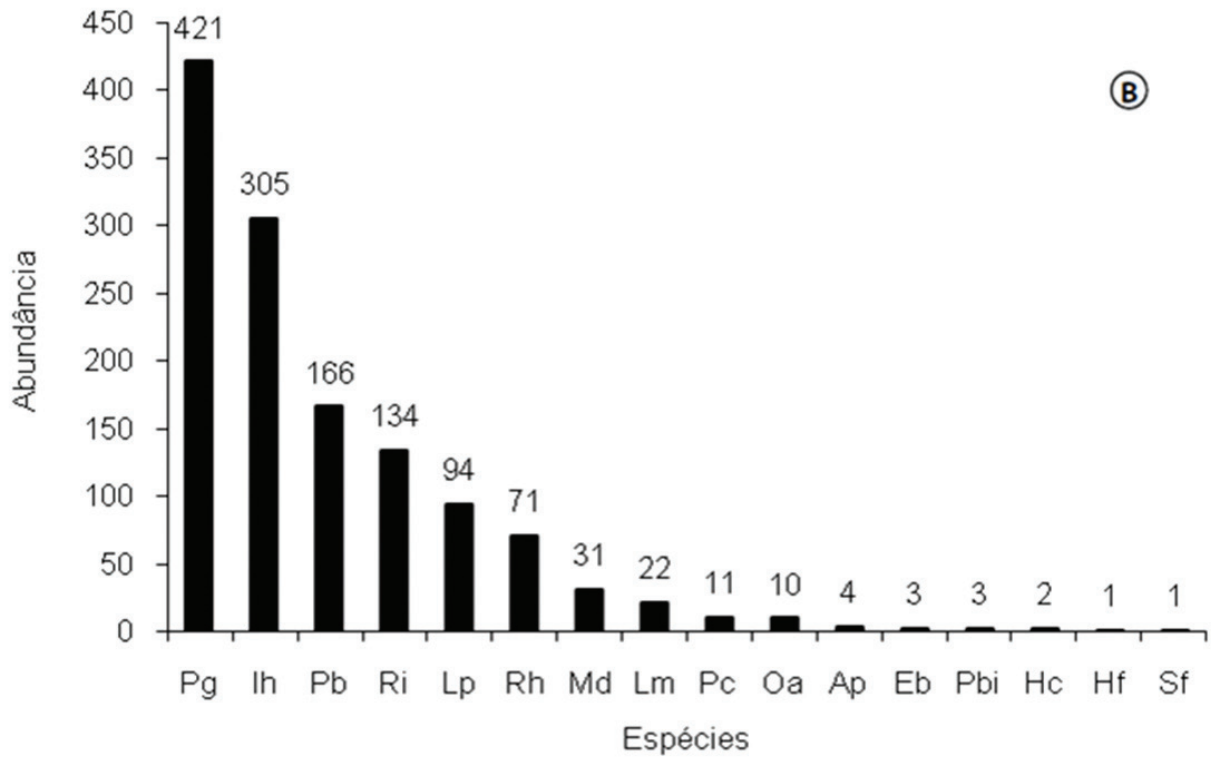

Figura 3. Abundância de anfíbios entre maio de 2008 a abril de 2010 no Parque Natural Municipal de Sertão, RS, em dois ambientes: A) borda; B) interior. Acima de cada coluna é indicado o número total de indivíduos por espécie. Legenda: $\mathrm{Ap}=$ A. perviridis; $\mathrm{Eb}=$ E. bicolor $; \mathrm{Hf}=H$. faber; $\mathrm{Hc}=H$. curupi $\mathrm{Ih}=I$. henselli $\mathrm{Ll}=$ L.latrans $; \mathrm{Lm}=$ L. mystacinus $; \mathrm{Lp}=$ L. plaumanni $; \mathrm{Md}=$ M. devincenzii $; \mathrm{Oa}=$ O. americanus $; \mathrm{Pb}=P$. bigibbosa $; \mathrm{Pbi}=P$. biligonigerus $; \mathrm{Pc}=P$. cuvieri $; \mathrm{Rh}=R$. henseli $; \mathrm{Ri}=R$. icterica $; \mathrm{Sf}=S$. fuscovarius $; \mathrm{Sa}=S$. aromothyella $; \mathrm{Ss}=$ S. squalirostris .

Figure 3. Abundance of frogs in pitfall traps from May 2008 through April 2010 in the Parque Natural Municipal de Sertão, RS, in two environments: A) border; $\mathrm{B}$ ) interior. The number of individual each species is indicated above the bars. Legend: $\mathrm{Ap}=$ A. perviridis; $\mathrm{Eb}=$ E. bicolor; $\mathrm{Hf}=H$. faber; $\mathrm{Hc}=$ H.curupi; $\mathrm{Ih}=$ I. henselli $; \mathrm{Ll}=$ L. latrans $; \mathrm{Lm}=$ L. mystacinus $; \mathrm{Lp}=$ L. plaumanni $; \mathrm{Md}=$ M. devincenzii $; \mathrm{Oa}=$ O. americanus $; \mathrm{Pb}=P$. bigibbosa $; \mathrm{Pbi}=P$. biligonigerus; $\mathrm{Pc}=$ P. cuvieri $; \mathrm{Rh}=$ R. henseli $; \mathrm{Ri}=$ R. icterica $; \mathrm{Sf}=$ S. fuscovarius $; \mathrm{Sa}=$ S. aromothyella $; \mathrm{Ss}=$ S. squalirostris.

Tabela 3. Regressão múltipla entre abundância e riqueza de anfíbios e serpentes e as variáveis climáticas no Parque Natural Municipal de Sertão, no período de maio de 2008 a abril de 2010 .

Table 3. Multiple regression between abundance and richness of amphibians and snakes and climatic variables in the Parque Natural Municipal de Sertão from May 2008 through April 2010.

\begin{tabular}{ccccc}
\hline & Variáveis Climáticas & Coeficientes & AIC & P \\
\hline \multirow{2}{*}{ Abundância de anfíbios } & Temperatura mínima & 177,89 & 215,61 & 0,01 \\
& Temperatura máxima & $-151,99$ & 213,76 & 0,02 \\
\hline Abundância de serpentes & Umidade relativa do ar & $-1,42$ & 62,05 & 0,05 \\
\hline \multirow{2}{*}{ Riqueza de serpentes } & Umidade relativa do ar & $-0,77$ & 17,90 & 17,14 \\
& Temperatura média & 0,73 & $<, 01$ & $<, 01$ \\
\hline
\end{tabular}

AIC $=$ Critério de Informação de Akaike.

$\mathrm{AIC}=$ Akaike Information Criterion . 

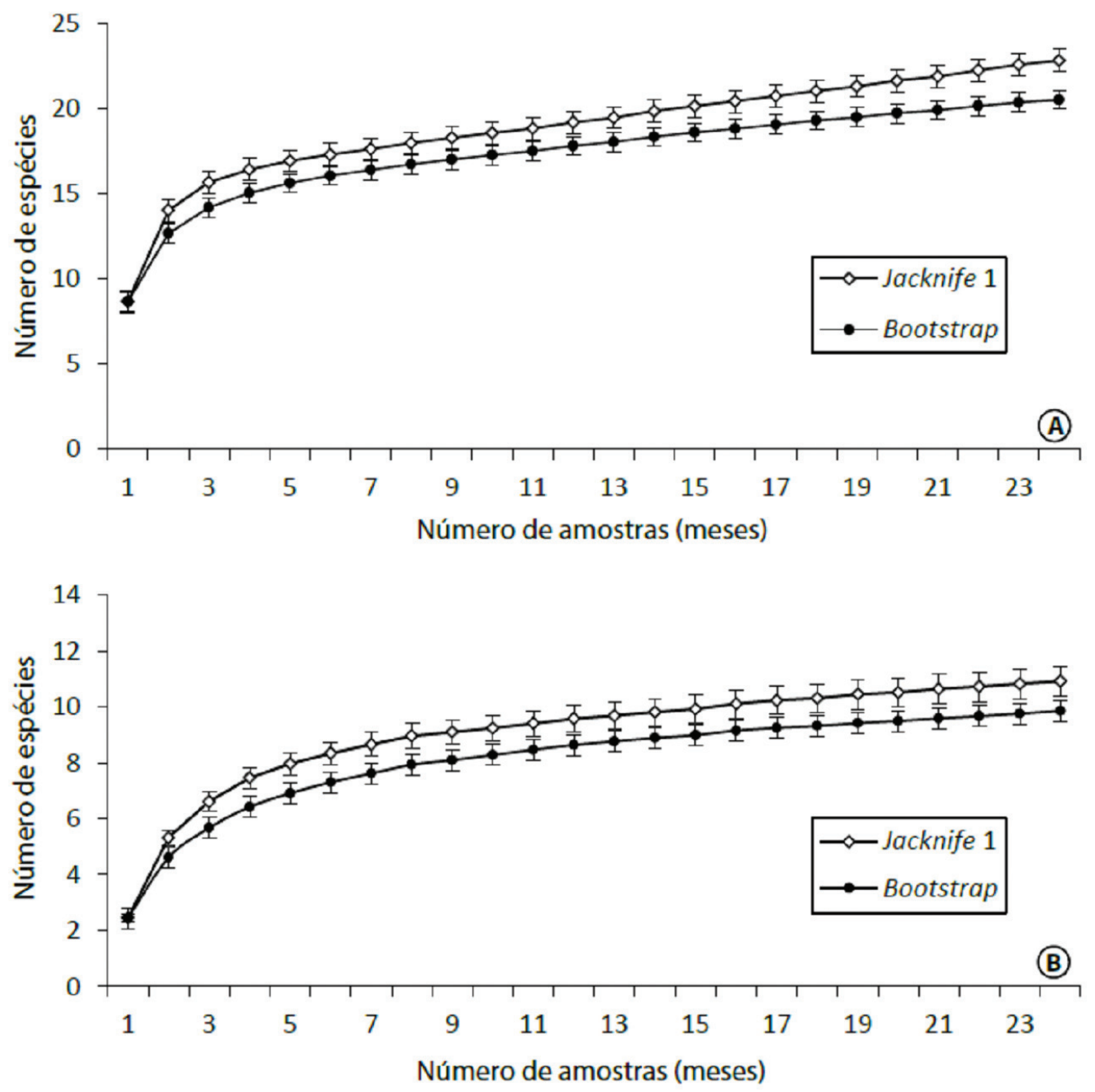

Figura 4. Curvas de rarefação para anfíbios (A) e répteis (B) geradas pelos estimadores Bootstrap e Jacknife em armadilhas de queda (pitfalls) no Parque Natural Municipal de Sertão, no período de maio de 2008 a abril de 2010.

Figure 4. Rarefaction curves for amphibians (A) and reptiles (B) generated by the Bootstrap estimators and Jacknife in pitfall traps in the Parque Natural Municipal de Sertão from May 2008 through April 2010.

contempla espécies arborícolas. Mesmo assim, foram amostradas cinco espécies de hilídeos (Aplastodiscus perviridis, Hypsiboas faber, H. curupi, Scinax aromothyella e S. fuscovarius). A presença dessas espécies nas armadilhas pode ter sido acidental, pois pode ter acontecido em períodos de chuva mais intensa o que ocasionaria a sua queda. A maioria das espécies está associada a ambientes florestados e quase todas as espécies já eram conhecidas para a região norte do estado do RS (Grando et al. 2004, Zanella et al. 2007a, b, Zanella \& Busin 2007), exceto H. curupi (Zanella et al. 2012) que constituí um novo registro para esta região do estado. Entretanto, a riqueza deste fragmento pode estar subestimada, pois não foram empreendidas muitas horas de busca ativa à noite, o que certamente elevaria o numero de espécies encontradas.

As espécies de répteis encontradas são típicas de áreas florestadas e abertas da Mata Atlântica. Salvator merianae foi encontrado tanto na área aberta como dentro do fragmento, pois apresenta grande capacidade de deslocamento entre os vários tipos de ambientes (Dixo \& Verdade 2006, Hartmann \& Giasson 2008). Bothrops cotiara, espécie associada a matas de araucária, que consta na lista das espécies ameaçadas do Rio Grande do Sul na categoria vulnerável (Di-Bernardo et al. 2003), foi encontrada somente nas armadilhas do interior do fragmento. Outro fator a ser analisado é a baixa semelhança entre o PNMS e outro estudo em Passo Fundo, distante $30 \mathrm{~km}$. O fragmento de Sertão, por ser maior que o de Passo Fundo, apresenta um número maior de espécies associadas à floresta. Estas diferenças provavelmente são consequência de fatores como o tamanho do fragmento, das alterações da estrutura da vegetação no interior da floresta, além das modificações causadas pela inserção de monoculturas no entorno do Parque. Apesar de não ter sido avaliado este parâmetro, o parque sofreu, nas últimas décadas, extração de madeira o que pode ter alterado a sua composição. Piroli \& Chaffe (2003) observaram, neste fragmento, a distribuição irregular das espécies florestais. Pontes et al. (2009) sugerem que a ausência de microhábitats potenciais para uso das espécies de serpentes em áreas florestadas para a termorregulação, forrageamento, abrigo e sítios de ovoposição, reduzem a riqueza e abundância de serpentes. A diferença na composição dessas comunidades pode ser um indício de que as alterações ambientais interferem na distribuição das espécies. Entretanto, outros fatores podem influenciar a riqueza encontrada na área, como o esforço de amostragem e os métodos 
utilizados. Em áreas florestais maduras e pouco perturbadas a fauna atinge um equilíbrio no número de espécies e nas relações entre riqueza e abundância (Scott 1976) e a equitabilidade é uma medida do grau de homogeneidade de distribuição da abundância de espécies em comunidades (Silva et al. 2011). Dixo \& Verdade (2006) verificaram que a composição da taxocenose de anuros de serrapilheira na Mata Atlântica do estado de São Paulo, entre as áreas de vegetação secundária e madura foi significativa, podendo estar associada a fatores biológicos das espécies e também ao estado de conservação das áreas estudadas. Moraes et al. (2007) observaram em dois ambientes de Mata Atlântica que a dominância de anfíbios observada na área preservada foi menor que na área alterada. Aárea de estudo apresenta menor equitabilidade de anfíbios na borda, seguindo o padrão encontrado por Cicchi et al. (2009) no sudeste do Brasil, onde as três espécies dominantes de anfíbios anuros representaram mais da metade $(55,5 \%)$ da amostragem e duas espécies de répteis representaram $56,4 \%$ do número de indivíduos coletados. Os dados encontrados, neste estudo, podem estar relacionados à interferência nos ambientes da borda, alterando suas características originais, e que suportam a ocorrência de espécies de áreas abertas e florestais. A maior homogeneidade com menores distúrbios antrópicos no interior do fragmento pode estar propiciando a menor dominância e maior equitabilidade das espécies florestais.

A espécie mais abundante de anuro, registrada em armadilhas de queda em ambos os ambientes foi Physalaemus gracilis. Esta espécie é conhecida por ser tolerante a alterações antrópicas (Vasconcelos et al. 2010) e ocorrer em áreas abertas (Kwet et al. 2010) ou áreas perturbadas. A presença desta espécie nos dois ambientes mostra a plasticidade ambiental existente em algumas espécies e, no interior do fragmento, onde a sua dominância foi menor, mostra um maior equilíbrio das espécies em ambientes com menor interferência antrópica. Em relação às serpentes, a espécie mais abundante na borda foi Atractus paraguayensis, indicando a sua preferência por áreas abertas (Hartmann \& Giasson 2008).

Armadilhas de queda foram mais efetivas na amostragem da riqueza de espécies de serrapilheira quando comparadas aos outros métodos, mesmo quando os dados mostram as coletas exclusivas por este método $(29,2 \%)$. Estudos mostram a eficácia destes métodos, especialmente de serrapilheira (Dixo \& Verdade 2006, Condez et al. 2009) e uma alta eficiência qualitativa das armadilhas de queda é geralmente registrada para anfíbios (Vasconcelos et al. 2010). Entretanto, inventários de herpetofauna devem abranger uma combinação de métodos para que se assegure um número maior de espécies (Condez et al. 2009). De fato, a curva de acumulação de espécies, neste estudo, não estabilizou e os estimadores de riqueza indicam que o número de espécies de anfíbios capturadas nas armadilhas de queda pode ser maior que o registrado. Por outro lado, os métodos utilizados foram pouco eficientes para amostrar os répteis, pois apresentaram baixa riqueza quando comparado a outros estudos já desenvolvidos no Rio Grande do Sul (Di-Bernardo et al. 2007) e somente Taeniophallus affinis constituiu um novo registro para a região (Paula et al. 2011). Estudos mostram que a densidade de répteis de áreas tropicais tem sido baixa quando comparado com anfíbios (Almeida-Gomes et al. 2008) e os nossos resultados concordam com esta tendência, pois o esforço nas armadilhas foi o mesmo para ambos os grupos. Répteis são menos abundantes e mais difíceis de serem amostrados que anfíbios (Araujo \& Almeida-Santos 2011). Entretanto, a curva de rarefação tendeu à estabilização, indicando que quase todas as espécies foram amostradas e as estimativas de aumento da riqueza pelos estimadores, mesmo pequena, é corroborada por Zanella \& Cechin (2006), que indicaram a presença de 14 espécies em área próxima. Entretanto, embora o esforço de amostragem tenha sido o mesmo para anfíbios e répteis não podemos deixar de levar em consideração que existem espécies que, por suas características biológicas, raramente são registradas, mesmo utilizando métodos complementares. Há, portanto, a necessidade de continuar os estudos na área para ampliar a amostragem da riqueza regional, principalmente de serpentes.

As maiores abundâncias de anfíbios foram encontradas quando as temperaturas mínimas eram maiores. As demais variáveis ambientais, com o modelo de regressão utilizado, não apresentaram associação com os anfíbios, indicando que as variáveis climatológicas, quando testadas em conjunto, explicaram muito pouco da riqueza e abundância das espécies. Na área de estudo não existe uma estação chuvosa definida, mas sazonalidade bem definida. Diversos estudos indicam que, em ambientes sazonais, a maioria das espécies tem atividade reprodutiva associada à estação chuvosa (Duellman 1978, Rossa-Feres \& Jim 1994, Toledo et al. 2003, Conte \& Rossa-Feres 2006, Moreira et al. 2008, Santos et al. 2008). Em um estudo, realizado na região central do RS, em área do Bioma Pampa, ocorreram correlações positivas fracas da riqueza de espécies com a precipitação mensal acumulada e a abundância com a temperatura média máxima, indicando que, naquela área, a temperatura parece atuar mais sobre a abundância de machos em atividade de vocalização e a precipitação sobre a riqueza (Santos et al. 2008). A ausência de associação, encontrada no presente estudo, pode indicar que quando as variáveis ambientais são analisados em conjunto, a temperatura parece atuar mais sobre a abundância de anfíbios que outros fatores abióticos.

A riqueza das serpentes foi influenciada pela temperatura, pois verificamos maior captura em estações mais quentes quando comparadas com épocas chuvosas. Variações na temperatura e pluviosidade exercem impacto sobre os padrões de atividade de serpentes (Gibbons \& Semlitsch 1987, Lillywhite 1987, DiBernardo et al. 2007). Em Passo Fundo, RS, a abundância de serpentes foi relacionada à temperatura máxima e não à pluviosidade (Zanella $\&$ Cechin 2009), indicando que a temperatura pode ser importante por limitar a atividade das serpentes no período mais frio do ano (Di-Bernardo et al. 2007).

Nossos resultados mostram a importância de áreas florestadas para a preservação das espécies e destacam a importância de inventários faunísticos para o melhor conhecimento da herpetofauna contribuindo com informações que poderão subsidiar estudos de conservação de áreas degradadas.

\section{Agradecimentos}

Os autores agradecem a Prefeitura Municipal de Sertão pela autorização para o desenvolvimento do trabalho no Parque. Ao Conselho de Pesquisa e Desenvolvimento (Cnpq) pelo auxílio financeiro. Ao Ibama/Sisbio pelas licenças concedidas (n. 145431/2009 e26826-1/2011). Agradecemos aos revisores anônimos pelas sugestões e leitura crítica do manuscrito. Ao Sr. Oldemar e D. Chica pelo uso da propriedade nos acampamentos da equipe e à comunidade do entorno do Parque.

\section{Referências Bibliográficas}

ALMEIDA-GOMES, M., VRCIBRADIC, D., SIQUEIRA, C.C., KIEFER, M.C., KLAION, T., ALMEIDA-SANTOS, P., NASCIMENTO, D., ARIANI, CV., BORGES-JUNIOR, VNT., FREITAS-FILHO R.F., VAN SLUYS, M. \& ROCHA, C.F.D. 2008. Herpetofauna of an Atlantic rainforest area (Morro São João) in Rio de Janeiro State, Brazil. Na. Acad. Bras. Cienc. 80(2):291-300.

ARAUJO, C.O. \& ALMEIDA-SANTOS, S.M. 2011. Herpetofauna in a cerrado remnant in the state of São Paulo, Southeastern Brazil. Biota Neotrop. 11(3): http://www.biotaneotropica.org.br/v11n3/en/abstract ?article +bn00511032011 
ARMSTRONG, C.G. \& CONTE, C.E. 2010. Taxocenose de anuros (Amphibia:Anura) em uma área de Floresta Ombrófila Densa no Sul do Brasil. Biota Neotrop. 10(1): http://www.biotaneotropica.org.br/v10n1/ en/abstract?article+bn00610012010

ÁVILA-FERREIRA, R.W. \& FERREIRA, V.L. 2004. Riqueza e densidade de vocalizações de anuros (Amphibia) em uma área urbana de Corumbá, Mato Grosso do Sul, Brasil. Rev. Bras. Zool. 21(4):887-892. http://dx.doi. org/10.1590/S0101-81752004000400024

BÉRNILS, R.S. \& H.C. COSTA (org.). 2011. Brazilian reptiles - List of species. http://www.sbherpetologia.org.br/. Sociedade Brasileira de Herpetologia (último acesso em 05/07/2012)

BERTOLUCI, J.A., BRASSALOTI, R.A., RIBEIRO JUNIOR, J.W., VILELA, V.M.F.N. \& SAWAKUCHI, H.O. 2007. Species composition and similarities among anuran assemblages of four forest sites in southeastern Brazil. Sci. Agric. 64(4):364-374. http://dx.doi.org/10.1590/S010390162007000400007

BERTOLUCI, J., CANELAS., M.A.S, EISEMBERG, C.C., PALMUTI, C.F.S \& MONTINGELLI, G.G. 2009. Herpetofauna of Estação Ambiental de Peti, an Atlantic Rainforest fragment of Minas Gerais State, southeastern Brazil. Biota Neotrop. 9(1): http://www.biotaneotropica.org.br/v9n1/en/ abstract?inventory+bn01409012009

BOTH, C., KAEFER, I.L., SANTOS, T.G. \& CECHIN, S.T.Z., 2008. An austral anuran assemblage in the Neotropics: seasonal occurrence correlated with photoperiod. J. Nat. Hist. 42:205-222. http://dx.doi. org/10.1080/00222930701847923

CECHIN, S.Z. \& MARTINS, M. 2000. Eficiência de armadilhas de queda (pitfall traps) em amostragens de anfíbios e répteis no Brasil. Rev. Bras. Zool. 17(3):729-740. http://dx.doi.org/10.1590/S010181752000000300017

CICCHI, P.J.P., SERAFIM, H., SENA, M.A., CENTENO, F.C. \& JIM, J. 2009. Herpetofauna em uma área de Floresta Atlântica na Ilha Anchieta, município de Ubatuba, sudeste do Brasil. Biota Neotrop. 9(2): http://www. biotaneotropica.org.br/v9n2/en/abstract?inventory+bn01009022009

COLWELL, R.K. \& CODDINGTON, J.A. 1994. Estimating terrestrial biodiversity through extrapolation. Phil. Trans. Royal Soc. London B, 345:101-118. PMid:7972351. http://dx.doi.org/10.1098/rstb.1994.0091

CONDEZ, T.H, SAWAYA, R.J. \& DIXO, M., 2009. Herpetofauna of the Atlantic Forest remnants of Tapiraí and Piedade region, São Paulo state, southeastern Brazil. Biota Neotrop. 9(1): http://www.biotaneotropica. org.br/v9n1/en/abstract?inventory+bn01809012009

CONTE, C.E., ROSSA-FERES, C. 2006. Diversidade e ocorrência temporal da anurofauna (Amphibia, Anura) em São José dos Pinhais, Paraná, Brasil. Rev. Bras. Zool. 23(1):162-175. http://dx.doi.org/10.1590/S010181752006000100008

DI-BERNARDO, M., BORGES-MARTINS, M. \& OLIVEIRA, R.B. 2003. Répteis. In Livro vermelho da fauna ameaçada de extinção no Rio Grande do Sul (C.S. Fontana, G.A. Bencke, \& R.E. Reis, eds). EDIPUCRS, Porto Alegre, p.165-188.

DI-BERNARDO, M., BORGES-MARTINS, M., OLIVEIRA, R.B. \& PONTES, G.M.P. 2007. Taxocenoses de serpentes de regiões temperadas do Brasil. In Herpetologia no Brasil II (L.B. Nascimento\& M.E. Oliveira, eds.). Sociedade Brasileira de Herpetologia, Belo Horizonte, p.222-263.

DIXO, M. \& VERDADE, V.K. 2006. Herpetofauna de serrapilheira da Reserva Florestal de Morro Grande, Cotia (SP). Biota Neotrop. 6(2): http://www. biotaneotropica.org.br/v6n2/pt/abstract?article+bn00706022006

DUELLMAN, W.E. 1978. The biology of an Equatorial Herpetofauna of Amazonian Ecuador. Misc. Publ. Univ. Kansas. 65:1-352.

DUELLMAN, W.E. 1999. Distribution patterns of amphibians in South America. In Patterns of distribution of amphibians: a global perspective (W.E. Duellman, ed). The Johns Hopkins University Press, Baltimore, p.255-328.

EMBRAPA. Centro Nacional de Pesquisa de Trigo - CNPT. Estação de agrometereologia. 2011. http//www.cnpt.embrapa.br/pesquisa/agromet (último acesso em 05/10/2012).
GIBBONS, J.W. \& SEMLITSCH, R.D. 1987. Activity patterns. In Snakes: Ecology and Evolutionary Biology (R.A. Seigel, J.T. Collins \& S.S Novak, eds.). MacMillan Publ. Co., New York. p.396-421.

GRANDO, J.V., GONÇALVES, F.A. \& ZANELLA, N. 2004. Composição e Distribuição Estacional dos Anuros de um Remanescente de Floresta Nativa em área Urbana no Município de Passo Fundo, RS. Acta Biol. Leopold. 26:93-100.

HARTMANN, P.A., GIASSON, L.O.M. 2008. Répteis. In A fauna das áreas de influência Usina Hidrelétrica Quebra-Queixo. (J.J. Cherem\& M. Kammers, eds). Habilis Editora, Erechim, p.111-124.

KWET, A., LINGNAU, R. \& DI-BERNARDO, M. 2010. Pró-Mata: Anfíbios da Serra Gaúcha Sul do Brasil. Brasilien-Zentrum, University of Tübingen, Germany. 148 p.

LILLYWHITE, H.B. 1987. Temperature, energetics and physiological ecology. In Snakes: Ecology and Evolutionary Biology. (R.A. Seigel, J.T. Collins, \& S.S. Novak, eds.). MacMillan Publ. Co., New York, p.422-477.

LUCAS, E.M. \& FORTES, V.B. 2008.Frog diversity in the Floresta Nacional de Chapecó, Atlantic Forest of Southern Brazil. Biota Neotrop. 8(3): http:// www.biotaneotropica.org.br/v8n3/pt/abstract? article +bn 00508032008

LUCAS, E.M. \& MAROCCO, J.C. 2011. Anurofauna (Amphibia, Anura) em um remanescente de Floresta Ombrófila Mista no Estado de Santa Catarina, Sul do Brasil. Biota Neotrop. 11(1): http://www.biotaneotropica. org.br/v11n1/en/abstract?article+bn01211012011

MAGURRAN, A.E. 1988. Ecological diversity and its measurement. Princeton, Princeton University, 179p. http://dx.doi.org/10.1007/978-94015-7358-0 http://dx.doi.org/10.1007/978-94-015-7358-0

MÄHLER JUNIOR, J.K.F. \& LAROCCA, J.F. 2009. Fitosionomias, desmatamento e fragmentação da Floresta com Araucária. In Floresta com Araucária: ecologia, conservação de desenvolvimento sustentável. (C.R. Fonseca,Souza, A.F. Leal-Zanchet, A.M. Dutra, T. Backes, A. \& Ganado G., eds.). Holos, Ribeirão Preto, p. 243-252.

MALTCHIK, L., PEIXOTO, C.D., STENERT, C., MOREIRA, L.F.B. \& MACHADO, I.F. 2008. Dynamics of the terrestrial amphibian assemblage in a flooded riparian Forest fragment in a Neotropical region in the south of Brazil. Braz. J. Biol. 4:763-769. http://dx.doi.org/10.1590/S151969842008000400010

MITTERMEIER, R.A., GIL, P.R., HOFFMANN, M., PILGRIM, J., BROOKS, J., MITTERMEIER, C.G., LAMOURUX, J. \& FONSECA, G.A.B. 2004. Hotspots revisited: earth's biologically richest and most endangered terrestrial ecoregions. Cemex, Washington.

MORAES, R.A., SAWAYA, R.J. \& BARRELA,W. 2007. Composição e diversidade de anfíbios anuros em dois ambientes de Mata Atlântica no Parque Estadual Carlos Botelho, São Paulo, sudeste do Brasil. Biota Neotrop. 7(2): http://www.biotaneotropica.org.br/v7n2/pt/ abstract?article+BN00307022007

MOREIRA, L.F.B., MACHADO, I.F., LACE, A.R.G.M. \& MALTCHIK, L. 2008. Anuran amphibians dynamics in an intermitent pond in southern Brazil. Acta Limnol. Bras. 20:205-212.

MYERS, N., MITTERMEIER, R.A., MITTERMEIER, C.G., DA FONSECA, G.A.B. \& KENT, J. 2000. Biodiversity hotspots for conservation priorities. Nature 403:853-858. PMid:10706275. http://dx.doi. org/10.1038/35002501

NARVAES, P., BERTOLUCI, J. \& RODRIGUES, M.T. 2009. Composição, uso do hábitat e estações reprodutivas das espécies de anuros da floresta de restinga da Estação Ecológica Juréia-Itatins, sudeste do Brasil. Biota Neot. 9(2): http://www.biotaneotropica.org.br/v9n2/en/ abstract?article+bn02009022009

PAULA, A., ZANELLA, N. \& GUARAGNI, S.A. 2011. Taeniophallus affinis (Günther, 1858) (Squamata, Serpentes, Dipsadidae): Distribution extension, Rio Grande do Sul, Brazil. Check List 7(3):285-286.

PIROLI, E.L. \& CHAFFE, P.P. 2003. Análise florística e determinação de volume das principais espécies ocorrentes em uma Floresta Ombrófila Mista. In Encontro de energia no meio rural, 3, Campinas: http://www. proceedings.scielo.br/scielo.phpscript $=$ sci_arttext\&pid=MSC0000000 022000000100001\&lng=en\&nrm=abn (último acesso em 05/07/2012). 
POMBAL JUNIOR, J.P. \& GORDO, M. 2004. Anfíbios Anuros da Juréia. In Estação Ecológica Juréia-Itains. Ambiente físico, flora e fauna. (O.A.V. Marques \& W. Duleba, org.). Holos Editora, Ribeirão Preto, p.243-256.

PONTES, J.A.L., PONTES, R.C. \& ROCHA, C.F.D. 2009. The snake community of Serra do Mendanha, in Rio de Janeiro state, southeastern Brazil: composition, abundance, richness and diversity in areas with different conservation degrees. Braz. J. Biol. 69(3):795-804. PMid:19802438. http://dx.doi.org/10.1590/S1519-69842009000400006

QUADROS, F.L. \& PILLAR, V.P. 2002. Transições floresta-campo no Rio Grande do Sul. Ciência e Ambiente. 24:109-118.

R DEVELOPMENT CORE TEAM. 2011. R: A Language and Environment for Statistical 3.Computing.R Foundation for Statistical Computing, Vienna, Austria.

ROSSA-FERES, D.C. \& JIM, J. 1994. Distribuição sazonal em comunidades de anfíbios anuros na região neotropical de Botucatu, São Paulo. Rev. Brasil. Biol. 54(2):323-334.

SANTOS, T.G., KOPP, K., SPIES, M.R., TREVISAN, R. \& CECHIN, S.Z. 2008. Distribuição temporal e espacial de anuros em área de Pampa, Santa Maria, RS. Iheringia, Sér. Zool. 98(2):244-253

SCOTT Jr., N.J. 1976. The abundance and diversity of the herpetofauna of tropical forest litter. Biotropica 8:41-58. http://dx.doi.org/10.2307/2387818

SEGALLA, M.V., CARAMASCHI, U., CRUZ, C.A.G., GARCIA, P.C.A., GRANT, T., HADDAD, C.F.B. \& LANGONE, J. 2012. Brazilian amphibians-List of species. Sociedade Brasileira de Herpetologia: http:// www.sbherpetologia.org.br (último acesso em 05/07/2012).

SILVA, R.A., MARTINS, I.A. \& ROSSA-FERES, D.C. 2011. Environmental heterogeneity: Anuran diversity in homogeneous environments. Zool. 28(5):610-618.

TABACHNICK, B.G. \& FIDELL, L.S. 2007. Using multivariate statistics. Pearson Education, Boston.
TOLEDO, L.F., ZINA, J. \& HADDAD, C.F.B. 2003. Temporal and Spatial Distribution in an Anuran Community in Municipality of Rio Claro, São Paulo, Brazil. Holos Environ. 3(2):136-149.

UNIVERSIDADE FEDERAL DO RIO GRANDE DO SUL - UFRGS. Laboratório de Herpetologia. 2010. versão 1.0. 2010. http://www.ufrgs. br/herpetologia (último acesso em 29/11/2012).

VASCONCELOS, T.S. \& ROSSA-FERES, D.C. 2005. Diversidade, distribuição espacial e temporal de anfíbios anuros (Amphibia, Anura) na região noroeste do estado de São Paulo, Brasil. Biota Neot. 5(2): http:// www.biotaneotropica.org.br/v5n2/pt/abstract?article+BN01705022005

VASCONCELOS, T.S., SANTOS, T.G., ROSSA-FERES, D.C. \& HADDAD, C.F.B. 2010. Similarity of ground-dwelling anuran (Amphibia) composition among different vegetation physiognomies in a Mesophytic Semideciduous Forest from southeastern Brazil. North-West. J. Zool. $6(2): 275-285$

ZANELlA, N., BUSIN, C.S., GIUSTI, A. \& CRESTANI, L. 2007 a. Amphibia, Anura, Bufonidae, Melanophryniscus devincenzii: First record for Brazil. Check List. 3:2.

ZANELLA, N., BUSIN, C.S. \& CRESTANI, L. 2007b. Eleutherodactylus henselii. Herp. Review. 38(4):474.

ZANELLA, N. \& BUSIN, C.S. 2007. Amphibia, Anura, Cycloramphidae, Proceratophrys bigibbosa: distribution extension for Rio Grande do Sul, Brazil. Check List. 3(1):65-66.

ZANELLA, N., BUSIN, C.S. \& MACHADO, L. 2012. Hypsiboas curupi: distribution. Herp. Review 43(3):440.

ZANELLA, N. \& CECHIN, S.Z. 2006. Taxocenose de Serpentes no Planalto Médio do Rio Grande do Sul, Brasil. Rev. Bras. Zool. 23(1):211-217. http://dx.doi.org/10.1590/S0101-81752006000100013

ZANELLA, N. \& CECHIN, S.Z. 2009. Influência dos fatores abióticos e da disponibilidade de presas sobre comunidade de serpentes do Planalto Médio, Rio Grande do Sul, Brasil. Iheringia, Zool. 99:111-114. http:// dx.doi.org/10.1590/S0101-81752006000100013

Recebido em 27/02/2013

Versão reformulada recebida em 10/10/2013

Publicado em 28/11/2013 